\title{
The Network Approach to General Intelligence*
}

\author{
Han L. J. van der Maas Alexander O. Savi Abe Hofman \\ Kees-Jan Kan Maarten Marsman
}

*The final version of this manuscript is published in General and Specific Mental Abilities: https://www . cambridgescholars.com/product/978-1-5275-3310-3 


\title{
CHAPTER FIVE
}

\section{THE NETWORK APPROACH TO GENERAL INTELLIGENCE}

\author{
HAN L. J. VAN DER MAAS, \\ ALEXANDER O. SAVI, ABE HOFMAN, \\ KEES-JAN KAN, \& MAARTEN MARSMAN
}

\section{Introduction}

The study of intelligence is a multidisciplinary endeavor. Progress has, for instance, been made in revealing genetic influences on intelligence (Plomin \& von Stumm, 2018), in identifying relevant processes in the brain (Jung \& Haier, 2007), and in artificial intelligence (AI). The recent successes with deep learning neural networks in AI are especially spectacular (Schmidhuber, 2015).

Despite this progress, it is undisputed that human intelligence is still a puzzling concept. There is no consensus on the definition of intelligence, not even within specific fields that study intelligence, such as psychometrics. Within the latter, there are, for instance, major disputes over whether intelligence is one thing (a general ability) or multiple things (Gardner, 1995). In our view, it is important to have a clear conception of what intelligence is. In this chapter we adhere to such a conception and present a formal model of psychometric intelligence. This model is admittedly overly simplistic, yet illuminates mechanisms that can explain some important and well replicated phenomena in the study of intelligence.

The model firstly acknowledges that the brain is a complex system (van der Maas, Kan, Hofman, \& Raijmakers, 2013). Complex systems are open systems consisting of many elements that interact non-linearly. Famous examples are ecosystems such as ant nests, weather phenomena such as hurricanes, and networks such as the internet. One remarkable feature is that such systems display self-organizing behavior: global 
regularities, or phenomena, emerge from the local interactions. The interdisciplinary study of complex systems has resulted in many insights, models, and techniques (Strogatz, 2018; Weisbuch, 2018). These results are evidently of importance for the study of brain functions, especially higher order cognitive functions. Somehow our brains, consisting of billions of neurons, display intelligent behavior: an emergent phenomenon that results from intricate local interactions.

Although complex systems research often requires a detailed mathematical model of the system under study, it is possible to model and explain certain empirical phenomena in a less exact way. An important example is the positive manifold in intelligence research. In 2006 van der Maas et al. proposed a completely new explanation of the positive correlations between cognitive tests, based on the idea of networks: a key modeling framework within complex system research. In this chapter we will a) explain the origin and setup of this so-called mutualism model, b) discuss criticisms that have been raised in response to the model, c) extend the model, d) present new statistical techniques for this model, and e) discuss new developments in the network modeling of general intelligence.

The network approach is developed in response to the dominant factor approach in the study of general intelligence. Some background on this factor approach and the study of general intelligence is important to understand the network approach to intelligence.

\section{The factor approach to general intelligence}

It is safe to say that the study of intelligence is the hallmark of differential psychology. The invention of intelligence testing, the development of factor analysis, and the theoretical model of mental power or $g$ have had an enormous influence on the study of individual differences in general. That is, when psychologists study individual differences in some psychological trait, they generally follow the same approach. They construct tests consisting of items clustered in sub-tests, collect data in some sample, perform factor analysis or employ some other latent trait approach, and arrive at the conclusion that differences in one or more latent traits explain observed individual differences. The justification of this approach, especially in intelligence research, mainly rests on its predictive power. Psychometric intelligence is a useful construct in personal selection and in the prediction of educational success (Deary et al., 2007).

But there is more to science than prediction. Outside differential psychology, in cognitive and developmental psychology for instance, 
researchers tend to ignore the field of intelligence research. On the one hand, this is somewhat understandable, as the $g$ model of intelligence does not provide an explanation of the 'mechanisms' underlying intelligent behavior. Intelligence is rarely incorporated in, or connected to, explanatory models of cognitive processes. This also holds for models of developmental processes (i.e. the basic $g$ model has no real account for the growth of cognitive ability). It is also unclear if $g$ itself develops and, if not, how intelligence develops (Ackerman \& Lohman, 2003). In this sense, the $g$ model is an anti-cognitive, anti-developmental model.

On the other hand, the simple neglect of the concept of general intelligence, its empirical basis, and its predictive power, is not satisfactory. The pattern of positive correlations between scores on a wide variety cognitive tests, the positive manifold, is a very robust phenomenon. It is one of the most replicated findings in psychology (Jensen, 1998). Intelligence tests are probably the best (and most controversial) tests the field of psychology has developed (Eysenck, 2018).

The division between the study of intelligence on the one hand, and the studies of cognition and cognitive development on the other hand, illustrates the timely relevance of Cronbach's famous division of scientific psychology into two disciplines, that of mechanisms and that of individual differences (Cronbach, 1957). Journals such as Intelligence, Structural Equation Modeling, Learning and Individual Differences on the one hand, and journals such as Cognition, Learning, Memory \& Cognition, Brain, and the Journal of Mathematical Psychology on the other hand, represent very different approaches to essentially the same problem. Cronbach was somewhat optimistic about the unification of these two disciplines, but 60 years later the division is still prominent.

We think one explanation of this state of affairs is the $g$ factor model itself. The main problem with the factor approach lies in the theory. It is simply unclear what $g$ is. It is remarkable that in thousands of publications on factor models of general intelligence this essential question is ignored. Some researchers take a practical point of view and just highlight the predictive value of $g$ (Gottfredson, 1998). Others take a statistical point of view and argue that they are not obliged to take a position about the ontological status of latent variables (Jonas \& Markon, 2016). We do not think these answers are satisfying. A theoretically relevant application of the latent variable model requires an answer to questions about the origin and causal nature of individual differences, hence of the latent variable, but there is no consensus at all on the cognitive or biological basis of $g$ (Ackerman, Beier, \& Boyle 2005). 
This state of affairs inspired us to look for new explanations of the positive manifold of correlations between cognitive tests. As said, the positive manifold is a robust phenomenon. Since the $g$ model, in whatever variant, is often conceived of as the only possible explanation of this phenomenon, the positive manifold is seen as evidence for $g$. However, at least two completely different kind of formal explanations exist. One explanation, mutualism theory, is relatively recent and the focus of this chapter. The other one, sampling theory, has been out there for a century, largely ignored, but recently re-introduced.

\section{Sampling theory}

It is important to understand sampling theory. Bartholomew, Deary, and Lawn (2009), and more recently Kovacs and Conway (2016) reintroduced the sampling theory of general intelligence, originally advocated by Thorndike (1927) and Thomson (1951). In these models the positive correlations between test scores are due to the fact that any two cognitive tests always share some underlying basic processes (or bonds). That is, cognitive tests are insufficiently specific. The overlap in shared processes will necessarily result in positive correlations between tests. Bartholomew, Deary, and Lawn (2009) generalized Thompson's (1951) model to account for multiple latent factors. Kovacs and Conway (2016) proposed a more elaborate version of sampling theory in order to account for the effects of domain-general executive processes, identified primarily in research on working memory, as well as more domain-specific processes. Although we think that both approaches are unclear in some important respects (Kan, van der Maas, \& Kievit, 2016), we do believe sampling is part of the explanation of the positive manifold.

A sampling type of explanation has also been repeatedly proposed for the relation between genes and phenotypes. For instance, Cannon and Keller's (2006) watershed model describes how specific genes influence 'upstream' endophenotypes. Kievit et al. (2016) extended this model to the domain of fluid intelligence. Another example is Anderson's (2001) model of the relation between genes and $g$.

\section{Mutualism}

Mutualism or interspecific cooperation is a biological concept like predation and parasitism, and is an important mechanism in the evolution of ecosystems. The mutualism model of general intelligence explicitly takes the ecosystem as a metaphor for our cognitive system. We can, for 
instance, think of our cognitive system as a lake. In explaining the positive manifold of correlations between cognitive tests, we could think about the differences between lakes.

Suppose we investigate the quality of several small lakes in say Europe. We set out to determine how well these ecosystems function, for instance regarding biodiversity. We start out to collect data concerning many different aspects of the lakes, such as measures of the quality of water, and the number and diversity of flora and fauna. Suppose we observe a positive manifold in these measures. In a factor analyses of such data we will observe a dominant general factor (Krijnen, 2004; van Bork et al., 2017). This would imply that 'good' lakes are better, to varying degrees, than 'bad' lakes in most measured aspects. This raises the question whether there is a $g$ factor for lakes. Is there an equivalent of mental power for ecosystems? We cannot exclude this possibility, but it is not how biologists think. They have other explanations of these phenomena in ecosystems.

In mathematical biology, ecosystems are often modeled as systems of coupled differential or difference equations, or more generally as networks. Famous is the Lotka-Volterra model for prey-predator population dynamics (May, 1973; Murray, 2002). This model generates a number of fascinating and complex phenomena, as described in any basic text on population dynamics. Current more realistic models can be very complex and detailed, providing accurate descriptions of, and predictions concerning, the dynamics of large ecosystems (e.g., Prakash \& de Roos, 2004). In these models, competitive and cooperative interactions are essential, and will give rise to correlations between different aspects or parts of the ecosystem.

This type of interactions in multivariate dynamical systems (see van Geert, 1991) forms the basis of our dynamical explanation of the positive manifold of cognitive performance. We argue that the positive manifold may be a by-product of the mutualistic interactions between different cognitive functions that occur during development. In our proposal, all functions or processes of the system are initially undeveloped and their traits are uncorrelated. During the development of the cognitive system, the dynamical interactions give rise to cognitive growth and to correlations among the various cognitive abilities.

\section{The mutualism model of intelligence}

The model of van der Maas et al. (2006) is the Lotka-Volterra mutualism model (Murray, 2002). It is based on a few assumptions. First it 
is assumed that the cognitive system consists of distinguishable parts or functions. There is clearly little consensus about the specific basic processes underlying intelligent behavior (Deary, 2002, p.153), but what these processes or functions exactly are is only of secondary importance. It is possible to adopt one theoretical framework, like the Sternberg (1988) model or the minimal cognitive architecture model of Anderson (1992), and develop the mutualism model in these terms. In general terms, our 'species' are cognitive abilities as they are for instance measured in intelligence tests. The second assumption is that each of these abilities undergoes auto-catalytic growth as the development of cognitive processes is largely an autonomous self-regulating process (Molenaar, Boomsma, \& Dolan, 1993). The third assumption is that this growth is constrained by limited resources such as neuronal speed and the size of neural systems associated with each of the cognitive processes (Garlick, 2002; Jensen 1998). For instance, short term memory reaches a maximum in late childhood, presumably because of limitations of the underlying neural system (e.g., Gathercole, 1999). The fourth assumption is that the limited resources are subject to both genetic and environmental influences. Assuming that these influences are additive suffices to explain several genetic effects in intelligence research.

The last major assumption is that cognitive abilities interact dynamically and that therefore cognitive abilities have mutual beneficial relations. Reciprocal causal relations are well known in the psychological literature. For instance, better short term memory stimulates developing better cognitive strategies, and better strategies increase the efficiency of short term memory (Siegler \& Alibali, 2002). Examples of positive influences of language on cognition, and visa versa, are syntactic bootstrapping (Fischer, Hall, Rakowitz, \& Gleitman, 1994) and semantic bootstrapping (Pinker, 1994). Other examples are the relations between cognition and meta-cognition (Sternberg, 1998), action and perception (Gibson, 1986), and performance and motivation (Dweck, 1986). Some recent papers confirm the existence of such mutualistic relations (e.g., Peng, Wang, Wang, \& Lin, 2018).

These relations might be weak but, as we shall see later, only small and sparse interactions are required to generate the positive manifold. We also expect that some of these relations operate via the social environment. Success in one task domain may lead to selection for certain trainings that benefit the task domain and other related domains. This is the idea behind the social multiplier effect model of Dickens (2007). This idea can be incorporated in the mutualism model without further assumptions (see van der Maas et al., 2017). 
It is possible that there are no facilitating interactions between certain processes, or even competitive or debilitating interactions. A simple example of the latter is the time constraint on cognitive expertise. Becoming an expert in say, music, may not allow other specializations. Van der Maas et al. (2006) demonstrated with computer simulations that the mutualism model can include a good degree of zero or competitive interaction, without affecting the fundamental result of the positive manifold of correlations.

Given these assumptions we can formulate the model:

$$
\begin{aligned}
\frac{d x_{i}}{d t} & =a_{i} x_{i}\left(1-\frac{x_{i}}{K_{i}}\right)+a_{i} \sum_{\substack{j=1 \\
j \neq i}}^{W} \frac{M_{i j} x_{i} x_{j}}{K_{i}} \text { for } i=1 . . W \\
K_{i} & =c_{i} G_{i}+\left(1-c_{i}\right) E_{i}
\end{aligned}
$$

Variables $x_{i}$ represent the $W$ cognitive abilities. Parameters $a_{i}$ are growth parameters, determining the steepness of the logistic growth function associated with each $x_{i}$. Parameters $K_{i}$ represent the limited resources of the logistic growth processes. The logistic growth function is in accordance with the assumption of auto-catalytic growth. The matrix $\boldsymbol{M}$ contains the interactions $M_{i j}$, used to specify the, possibly asymmetric, relations between pairs of abilities in development. $\boldsymbol{K}$ itself is a weighted (by $c_{i}$ ) sum of genetic and environmental contributions. The parameters $\boldsymbol{x}_{\boldsymbol{0}}$ (initial values), $\boldsymbol{a}$, and $\boldsymbol{K}$ differ over subjects, whereas the matrix $\boldsymbol{M}$ contains population parameters, equal for all subjects.

Each ability starts its growth process at a low value and follows a logistic growth curve until an asymptote (larger than $K_{i}$ ) is reached. At any point in time $t$ we can take a measurement of $\boldsymbol{x}$. By rerunning the model a number of times $(n)$, we collect data for $n$ subjects on $W$ cognitive abilities measured at time $t$. This matrix of data can be subjected to correlational and factor analysis.

Three scenarios were investigated in van der Maas et al. (2006): 1) all $K_{i}$ are uncorrelated and all $\left.M_{i j}=0,2\right)$ all $K_{i}$ are correlated and all $M_{i j}=0$, and 3) all $K_{i}$ are uncorrelated and all $M_{i j}=.05$. In the first scenario, we found no correlations between the $x_{i}$, as expected. The second scenario is in concordance with the $g$-factor case, as the limited resources do share some common source of variance. In this case we found the expected positive manifold. The third scenario represents the mutualism case. In this case, we also found the positive manifold, even though all individual parameters were uncorrelated, and hence the positive interactions produced the positive manifold. 


\section{Other simulation results}

Van der Maas et al. (2006) investigated whether the mutualism model could explain other phenomena in intelligence research, without adding new assumptions. One interesting example is the form of $\boldsymbol{M}$. Initially all $M_{i j}$ were set to the same value (scenario 3 ), but it is more realistic if the interactions show some variation. If the interactions are sampled from a normal distribution with a mean of .05 and a standard deviation of .06, about $20 \%$ of the interactions are expected to be negative. Such an $\boldsymbol{M}$ leads to correlation matrices that require more complex factor models. It was shown that the correlations were well described by higher-order factor models. These higher-order factor models are the standard in current factor models of actual intelligence data.

Another conclusion of this simulation is that the presence of the positive manifold requires only a few weak interactions in the mutualism model. This is important, since the empirical results on transfer between cognitive abilities often show no or weak effects, especially with regard to far-transfer (Schwaighofer, Fischer, \& Bühner, 2015). The literature on transfer is somewhat inconsistent, but transfer effects are often disappointing.

Additionally, we studied several developmental effects. First, the low predictive validity of test performance during infancy and early childhood (Honzik, 1983; McCall \& Carriger, 1993) easily follows from the model. Second, the model also explains the increase in heritability during development (Bartels, Rietveld, van Baal, \& Boomsma, 2002; Fulker, DeFries, \& Plomin, 1988; Haworth et al. 2010). Initially, the values of the variables $\boldsymbol{x}$ are determined by the growth parameters $\boldsymbol{a}$. The influence of $\boldsymbol{K}$ is low in this phase. Only later in development, when variables $\boldsymbol{x}$ reach their asymptotic values, $\boldsymbol{K}$ comes into play, and thus the genetic influences on $\boldsymbol{K}$.

\section{Criticism}

Van der Maas et al. (2006) mention a number of limitations. First, one can ask whether the mutualism model is really different from the factor model and sampling theory. In our view, although all these models explain the positive manifold equally well, they are conceptually very distinct. In the $g$-factor model the correlations are produced by a common source of cognitive performance in many domains. The $g$-factor is understood as a so-called reflective latent variable. In the network model there is no common source; the positive manifold is produced by the network structure. The statistical $g$-factor on the other hand, is interpreted as a 
formative variable; an index of the general quality of the cognitive system, akin to economic indexes such as the Dow Jones Industrial Average. Also in sampling theories the statistical $g$-factor should be interpreted as a formative variable. Yet test sampling theory and the mutualistic network model are very different. In test sampling theory the positive manifold is essentially a measurement problem. If we would be able to construct very specific tests, targeted at the single basic processes, the overlap in measurement would disappear, and so would the correlations between tests. In the mutualism model the correlations are real, created during development, and will not disappear when our IQ tests become more specific.

Second, the mutualism model does not constitute a parsimonious explanation of the data, in the sense that $\boldsymbol{M}$ may contain many parameters, we agree, but to our defense, we do not assume the presence of an unnecessary, rather mysterious latent variable. With respect to the number of entities (explanatory variables), the $g$ model is the more expensive model. Moreover, $\boldsymbol{M}$ can be sparse and still give rise to a positive manifold. Many kinds of restrictions may be placed on the elements in the matrix $\boldsymbol{M}$, which can be based on theoretical considerations, or the results of experimental studies. To explain the positive manifold, it suffices to assume $M_{i j}=\mathrm{c}$ for all subjects.

Third, the model is extremely simplistic. To name a few aspects, $\boldsymbol{M}$ most likely differs between subjects, not all growth processes will start at the same moment, and the linear model for the genetic and environmental impact disregards interactions. However, an advantage is that this model can easily be investigated using simulations and formal proofs. Furthermore, the simple mutualism model does explain key phenomena in intelligence research.

Fourth, the mutualism model in its original form cannot explain the socalled Jensen effect without additional assumptions. The Jensen-effect refers to the correlation between the vector of $g$-loadings of cognitive tests and the heritability coefficients of these tests, using the method of correlated vectors. Jensen (1998) computed, for a number of data sets, such correlation, which averaged about .5. This is taken as evidence for a biological and genetic interpretation of $g$. However, this interpretation is problematic. Kan, Wicherts, Dolan, \& van der Maas (2013) have shown that the heritabilities and $g$-loadings are higher for crystallized tests (such as vocabulary and arithmetic) than for typical fluid tests. This finding runs against $g$ theory. Furthermore, the extended mutualism model proposed in van der Maas et al. (2017) is able to explain this empirical pattern. 
Some authors added other points of criticism. Nisbett et al. (2012) mention that the mutualism model does not distinguish between genetic and environmental effects and integration with Dickens' (2007) model could provide a more compelling description of the development process. This is partly incorrect (see equation 2), but we agree that the multiplier effect is important. In van der Maas et al. (2017) it is explained how such integration could be established by a redefinition of $\boldsymbol{M}$. That is, one $x_{i}$ is used to represent the cognitive environment, which effectively incorporates the multiplier effect underlying Dickens' model.

Based on several data sets, Gignac (2014) claims that the $g$-factor is stable from 3 years on, which he sees as an indirect rejection of the mutualism model. This was recently replicated by Shahabi, Abad, and Colom (2018) with data in the age range of 6 to 12. In general, we think the literature on age (de-)differentiation is rather inconclusive due to various methodological problems (van der Maas, et al. 2006). In most studies, the first years of development are ignored. Also, the mutualism model does not specify the age at which the statistical $g$-factor should become stable. Also note that it is unclear what the $g$-theory predicts. According to Gignac, $g$-factor theory may be suggested to predict the strength of the $g$ factor to be largely constant across all ages, because this theory postulates biological and genetic substrates for $g$. But the biological substrate is all but constant in childhood, and $g$-theory is unspecific with regard to such developmental issues. At least a formal derivation of a prediction on the change in the strength of $g$ is missing.

Gignac (2016) also criticizes the mutualism model. He reasons that it would be very unlikely that the pattern of mutually beneficial interactions between cognitive abilities across individuals arise precisely in a manner that their latent variable inter-associations can be accounted for by a single latent variable. Van der Maas and Kan (2016) provided a rebuttal to these criticisms, showing that Gignac's main premise is incorrect.

In our current evaluation of the model we raise a new limitation of the model. From a developmental point of view the model makes some questionable assumptions. All the nodes and connections are available from birth, and only the values of $\boldsymbol{x}$ change. Below we will discuss the possibility to use growing networks as models of cognitive development.

\section{The extended 'unified' mutualism model}

Van der Maas et al. (2017) summarize the various extensions that have been proposed over the years. Mutualism remains the core of this model. The addition of a node that represents the environment incorporates 
Dickens multiplier idea. Cattell's investment theory can be represented by asymmetric relations in the interaction matrix $\boldsymbol{M}$. Furthermore, some fluid processes may be more central than others, reflected by stronger connections to other nodes. Such central processes, for instance working memory, will then correlate strongly with the statistical $g$-factor. All these extensions can be included into a unified network model with the specification of $\boldsymbol{M}$. We also think that sampling is part of the explanation of the positive manifold. Our current tests of intellectual functions clearly lack specificity (Lumsden, 1976). Our 'unified' model is shown in Figure $5-1$.

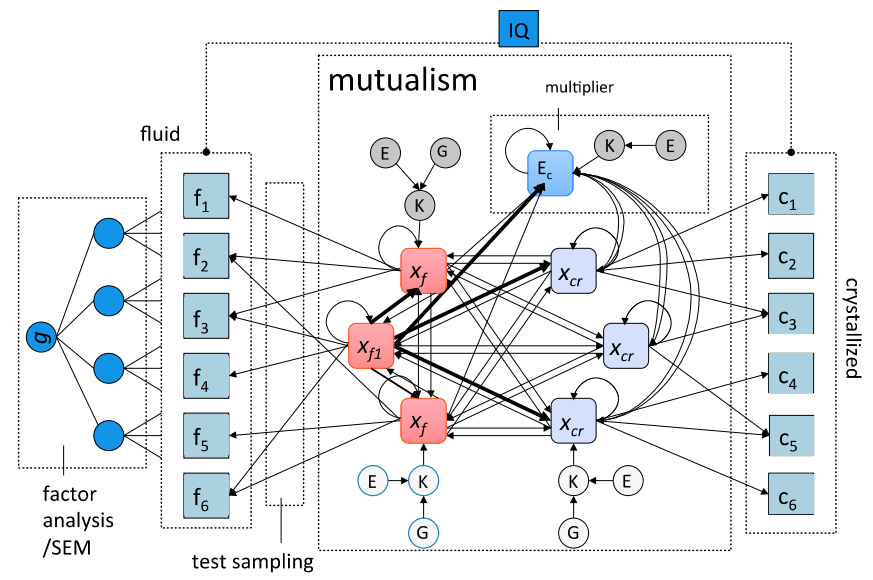

Figure 5-1. The unified model of general intelligence includes test sampling, reciprocal effects (both mutualistic and multiplier), and central cognitive variables (such as working memory capacity, $x_{f l}$ ). The $\mathrm{x}_{\mathrm{f}}$ and $\mathrm{x}_{\mathrm{c}}$ nodes represent fluid and crystalized cognitive abilities in the intelligence network. The $f_{i}$ and $c_{i}$ represent test scores for these abilities, the sum of which is IQ. The $g$-factor can be extracted using factor analysis on $\mathrm{f}$ (and $\mathrm{c}$ ) tests. 


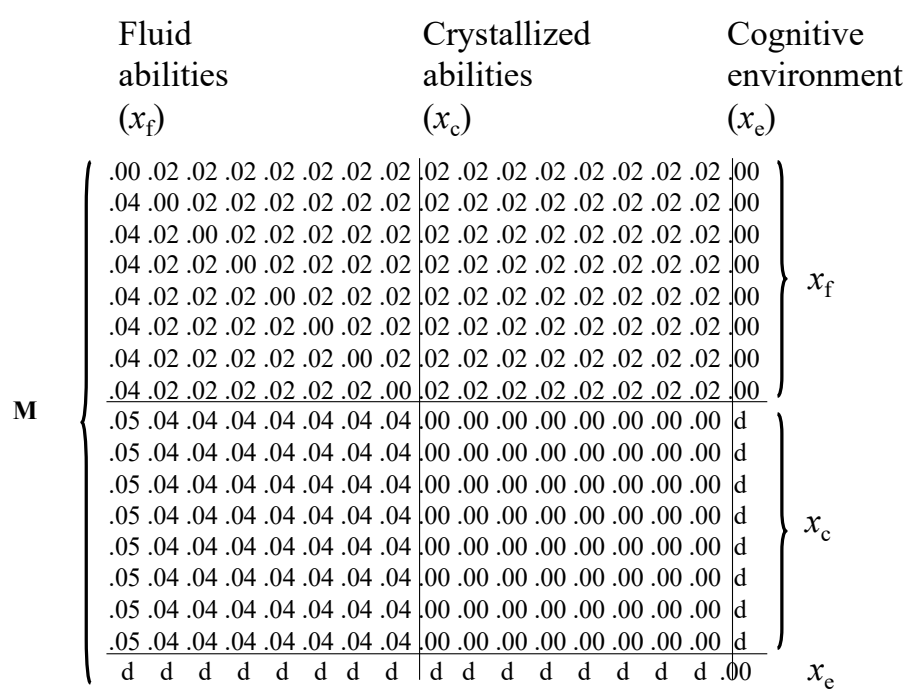

Figure 5-2. $M$ matrix for the unified model in Figure 5-2.

With this extended model most limitations of the basic mutualism model are resolved. However, the last limitation we mentioned requires a very different type of network model that we will describe in the last section.

\section{Fitting the model to data}

Much has been said on the relation between factor and network models. One confusing and equally fascinating fact, is that in both the factor approach and the network approach we can perform factor analysis, with similar statistical results. The difference lies purely in the interpretation of the statistical factor. In our view, the difference between the reflective and formative interpretation of the statistical latent variable is of fundamental importance (Edwards, \& Bagozzi, 2000).

In the reflective model, the latent variable is the common cause of the scores on the observed variables (Figure 5-3). A good example is the thermometer. Suppose we have a set of thermometers of mediocre quality. When we collect sets of measurements in different locations, we can subject the data to factor analysis. The resulting factor will represent a physical realistic variable that is the source of the differences in measurement values on each of the thermometers across the locations. In 
contrast, in the formative model the latent variable is an index of the state of some complex system. Economic indices are excellent examples; they summarize an economic situation, rather than cause economic success. Another example is health. Health measures summarize the overall state of the body (Plsek \& Greenhalgh, 2001), rather than cause better health
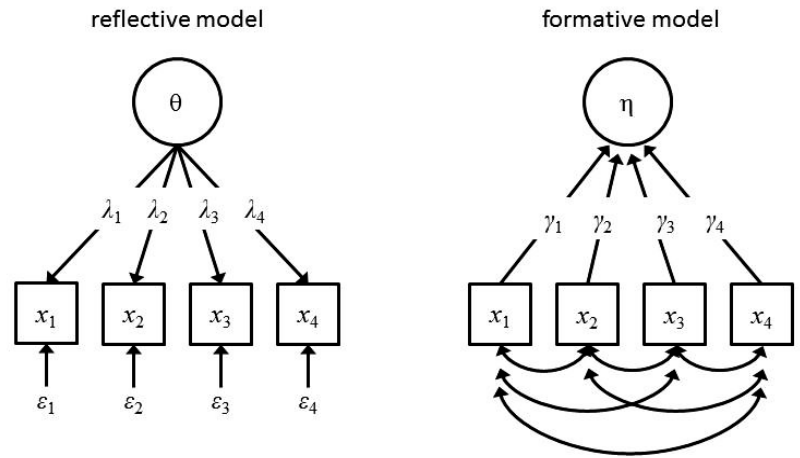

Figure 5-3. In the reflective model the latent variable (e.g., temperature) is the common cause of the manifest scores (e.g., thermometer values at different locations). In the formative model the latent variable (e.g., economical index) only summarizes the manifest scores (e.g., health index).

Standard structural equation models will thus not be decisive on the issue, but new possibilities have appeared recently. Since 2006, network science has become a productive area of research in psychology, with applications in especially clinical psychology. As in the mutualism model, the general hypothesis in psychological network models is that correlations between observed behaviors, such as cognitive abilities, psychopathological symptoms, or attitudes, are not due to unobserved common causes, but rather due to the network of interacting psychological and/or biological factors.

This approach has led to a number of new insights concerning, for instance, the co-morbidity of clinical symptoms (Cramer, Waldorp, van der Maas, \& Borsboom, 2010), early warnings for clinical disorders (van de Leemput et al., 2014) and the organization of attitudes (Dalege et al., 2016).

This work also led to new statistical analysis and visualization techniques (for an overview see Epskamp, Borsboom, \& Fried, 2018). The most popular models that are used for cross-sectional data are the Gaussian Graphical Model (GGM) for continuous data and the Ising model for binary data. Network changes can be estimated using vector- 
autoregression (VAR). A criticism of the validity of network psychometrics can be found in Forbes, Wright, Markon, \& Krueger (2017) with a reply in Borsboom et al. (2017).

For the mutualism model, the work of Kievit and colleagues (2017a, $2017 \mathrm{~b}$ ) is particularly relevant. They extended the latent change score model (LCSM) to bivariate LCSM (McArdle, 2001; McArdle et al., 2002) such that the relationship between the developments of abilities in two separate domains can be investigated.

$$
\begin{aligned}
& \Delta_{1, \mathrm{pt}}=\beta_{1} y_{1, p(t-1)}+\gamma_{21} y_{2, p(t-1)} \\
& \Delta_{2, \mathrm{pt}}=\beta_{2} y_{2, p(t-1)}+\gamma_{12} y_{1, p(t-1)}
\end{aligned}
$$

In this model, a change score $(\Delta)$ is defined for both domains for person $p$ over time $t$. The autocatalytic parameter, $\beta$, models the growth rate, with a positive $\beta$ indicating accelerating growth and a negative $\beta$ indicating decelerating growth. The coupling parameters $\gamma$ reflect the effect of the previous score in one domain (i.e., at time $t-1$ ) on the change in score in the other domain. By examining the $\gamma_{21}$ and $\gamma_{12}$ parameters, we can determine which domains influence the development of other domains. The idea is that the difference between a $g$-factor and mutualism model is reflected in the $\gamma$ parameters. This is because there is a clear relation between the $\gamma$ parameters in the LCSM and the $\boldsymbol{M}$ matrix in the mutualism model. This technique has now been applied to various datasets (Kievit et al. 2017a, 2017b; Kievit, Hofman, Nation, 2018, Hofman et al. 2018).

In all cases evidence for mutualistic coupling is reported. Psychometric network modeling can also be used as an exploratory means to help determine the number of factors in cognitive data (Golino and Demetriou, 2017). In Kan, van der Maas \& Levine (2018) we propose a more confirmatory approach to test competing network and factor analytic theories of intelligence against each other. The analysis requires the computation of a Gaussian graphical model of the full partial correlation matrix, which results in a saturated network model. Subsequently, by means of a graphical lasso procedure (Friedman, Hastie, \& Tibshirani, 2014), with the built-in tuning parameter ('lambda min ratio') procedure and the extended Bayesian information criterion, the network model is adjusted for the presence of false positive edges (Epskamp et al., 2012). This results in a non-saturated sparser network model (the 'glassoed network'). Once such model is implemented in the SEM framework and compared to competing models, we may consider the procedure as an example of 'the exploratory mode' of confirmatory modeling (Raykov \& 
Marcoulides, 2006). Ideally, in a true confirmatory analysis, one would specify the network a priori.

The glassoed network can be compared with the usual indices (AIC, BIC) with factor models, such as the higher-order factor model and the bifactor model. In a simulation study, Kan, van der Maas \& Levine (2018) demonstrate that this approach does not lead to false positives in favor of the glassoed network. Next, they investigated several empirical data-sets and found that the mutualism model generally provides a more satisfactory solution than previous established factor models.

\section{Wired cognition}

As mentioned before, the dynamics of the (extended) mutualism model are limited. All cognitive abilities $\boldsymbol{x}$ and connections $\boldsymbol{M}$ are there from the beginning, and only the values of $\boldsymbol{x}$ change. Although an improvement over $g$ theory, we think this limitation of the mutualism model requires attention. Therefore, we built the framework of a new theory, in which nodes and connections are dynamically added to the cognitive network (Savi, Marsman, van der Maas, \& Maris, 2018).

This wired cognition model starts with some rudimentary skills or knowledge, represented by just a few nodes. Over time, it grows to a well connected, extended network of cognitive skills. An example would be arithmetic. Toddlers may know some numbers and perhaps can count to ten. In this situation, the nodes are unstable and the connections are weak. Over time they learn more facts and arithmetic operations, and nodes and connections are added to their cognitive networks. The growth of these networks can be modeled by means of, for instance, a preferential attachment rule (Barabási \& Albert, 1999). In this way a semantic network can develop with clusters for specific sub-abilities like multiplication. One advantage of this model is that it relates intelligence to education. Teachers help students building their networks of expertise.

In Savi et al. (2018) we studied a very specific network, the FortuinKasteleyn (FK) network model (Fortuin, \& Kasteleyn, 1972). This model allows a formal derivation of developmental properties. It can be shown analytically that an FK model gives rise to the positive manifold. Similar to the mutualism model, this does not require a latent factor. And importantly, the genetics, the environment, and the development of a person are explicitly captured in the cognitive network, by means of the initial network structure, the gradual addition of nodes and edges to the network, and a growth mechanism. The model succeeds in describing 
individuals by unique cognitive networks, making it a truly idiographic model.

Future work will include the effect of forgetting, as weakly connected or unconnected nodes are probably prone to be forgotten. A toddler may keep the fact that $5+5=10$ in memory for some time, but if this fact is not connected to other knowledge, it might be forgotten soon. Additionally, weakly connected networks allow for sub clusters with incorrect knowledge (misconceptions) or deficient skills. This work is part of a grander goal to understand the growth (and decline) dynamics of a developing intelligence.

\section{Discussion}

Since the introduction of the mutualism model, network psychometrics and network modelling are rapidly gaining ground in psychology. Conceptualizing psychological traits and abilities as networks of interacting components has become a serious alternative for latent variable modelling. Current applications of network modelling range from psychopathology, attitudes (Dalege, Borsboom, van Harreveld, \& van der Maas, 2019), personality (Costantini et al., 2015), interest (Sachistal, 2018), and intelligence. In the field of psychopathology, the range of applications is especially impressive. Recent work concerns depression, anxiety disorders, post-traumatic stress, complex bereavement, autism, psychotic disorders, substance abuse, the general structure of psychiatric symptomatology, diagnostic manuals, health-related quality of life, and personality traits (for an overview and references, see Borsboom, 2017; Borsboom, Robinaugh, Psychosystems Group, Rhemtulla, \& Cramer, 2018).

In the last 10 years, we developed and extended our unified mutualism model that incorporates several additional explanatory mechanisms. We distinguish between fluid and crystalized abilities, added test sampling, and allow for a multiplier effect via the environment, which is important to explain various gene-environment correlation effects (Kan, Wicherts, Dolan, and van der Maas, 2013). These extensions are easily incorporated in the basic model. We also accept the idea that some abilities are more central than others. For instance, working memory may be more central than other abilities and therefore display a higher $g$-loading in statistical analyses. In van der Maas et al. (2017), we argued that network models can give rise to both continuous and discontinuous developmental change. The network approach thus also relates categorical and continuous latent variable models of individual differences. 
This extended mutualism model is still very different from $g$-theory. In the network model the statistical main factor is just an index of the state of the network and not a causal factor itself. In our view, the difference between the formative and reflective interpretation of the statistical $g$ factor is of utmost importance and not only for theoretical reasons.

First, if the mutualism model is correct, the search for a gene or brain area "for $g$ " will be of limited value. By assuming that the uncorrelated capacities K's are partly genetically influenced the mutualism model explains both the high heritability for general intelligence found in twin studies, as well as the increase of this heritability over time (see van der Maas et al., 2006). The recent successes in finding genome sequence differences accounting for significant contributions to the heritability of intelligence (for an overview, see Plomin \& von Stumm, 2018) does not change the picture. Such results cannot prove that $g$ is a biological causal entity as they are completely in line with the prediction of the mutualism model (and also sampling theory). Also, the biological unitary $g$ factor is perhaps inconsistent with Plomin and Stumm's conclusion that the genetic effects on intelligence are extremely pleiotropic and that hundreds of thousands of SNP associations are needed to account for the $50 \%$ heritability estimated by twin studies.

Secondly, there are implications for measurement (van der Maas, Kan, $\&$ Bosboom, 2014). A crucial difference between reflective and formative models concerns the role of the indicator variables (items or subtests). In the $g$-model, a reflective model, these indicators are exchangeable. New tests, with different factor loadings, could be added to a test battery without changing the essence of the measured $g$-factor. In formative models, however, indicators are not exchangeable. What we put in the index is a choice that rests on pragmatic grounds, for instance in its predictive value. The best IQ test is simply the combination of tests that best predicts educational or job success. When a certain cognitive capacity, say computational thinking, is valued to a greater extent in the current society, intelligence tests should be adapted to reflect individual differences therein.

The wired cognition model that we introduced in this paper can be seen as a successor of the mutualism model of general intelligence. Both are network models inspired by formal modeling approaches in the natural sciences. However, the wired cognition model is not an extension of the mutualism model; it differs in too many respects. In the mutualism model the architecture of the network is fixed, all nodes and edges (basic processes) are there from the beginning. The wired cognition model on the other hand, is a model of a developing network. 
Both models share two important ideas. The first is that models of individual differences should be based on models of the individual. With the network model we intend to bridge two separate research traditions, on the one hand the experimental research on cognitive mechanisms and processes, on the other hand the psychometric research on individual differences in intelligence. Cronbach's (1957) famous division of scientific psychology in two disciplines is still very true for the fields of cognition and intelligence. In order to bring these fields together we developed a mechanistic model in the cognitive tradition with the aim to explain a set of well-established key phenomena in the research of individual differences.

The second shared idea is that a scientific theory should be formulated as a mathematical or computational model. In cognitive research, the largely verbal Piagetian and neo-Piagetian models have been succeeded by concrete computational information processing models, both symbolic and connectionist. The traditional factor models of general intelligence are different in that they are statistical models of individual differences. They do not specify a (formal) model of intelligence in the individual. Sampling models and mutualism model have been formulated mathematically. The advantages are that models have to be precisely defined, prediction can be derived unambiguously and unexpected and undesirable by-effect of the model can be detected, for instance, in simulations.

\section{References}

Ackerman, P. L., \& Lohman, D. F. (2003). Education and g. In H. Nyborg (Ed.), The scientific study of general intelligence. Amsterdam: Pergamon.

Ackerman, P. L., Beier, M. E., \& Boyle, M. O. (2005). Working memory and intelligence: The same or different constructs? Psychological Bulletin, 131, 30-60.

Anderson, B. (2001). g as a consequence of shared genes. Intelligence, 29, 367-371.

Anderson, M. (1992). Intelligence and development: A cognitive theory. Oxford: Blackwell.

Barabási, A. L., \& Albert, R. (1999). Emergence of scaling in random networks. Science, 286(5439), 509-512.

Bartels, M., Rietveld, J. H., van Baal, G. C. M., \& Boomsma, D. I. (2002). Genetic and environmental influences on the development of intelligence. Behavior Genetics, 32(4), 236-249.

Bartholomew, D. J., Deary, I. J., \& Lawn, M. (2009). A new lease of life 
for Thomson's bonds model of intelligence. Psychological Review, $116(3), 567$.

Borsboom, D. (2017). A network theory of mental disorders. World Psychiatry, 16(1), 5-13.

Borsboom, D., Fried, E. I., Epskamp, S., Waldorp, L. J., van Borkulo, C. D., van der Maas, H. L., \& Cramer, A. O. (2017). False alarm? A comprehensive reanalysis of "Evidence that psychopathology symptom networks have limited replicability" by Forbes, Wright, Markon, and Krueger. Journal of Abnormal Psychology, 126(7), 989.

Borsboom, D., Robinaugh, D. J., Psychosystems Group, Rhemtulla, M., \& Cramer, A. O. (2018). Robustness and replicability of psychopathology networks. World Psychiatry, 17(2), 143-144.

Cattell, R. B. (1971). Abilities: Their structure, growth, and action. Oxford: Houghton Mifflin.

Costantini, G., Richetin, J., Preti, E., Casini, E., Epskamp, S., \& Perugini, M. (2017). Stability and variability of personality networks. A tutorial on recent developments in network psychometrics. Personality and Individual Differences, 136, 2019, Pages 68-78

Cramer, A. O. J., Waldorp, L. J., Van der Maas, H. L. J., \& Borsboom, D. (2010). Comorbidity: A network perspective. Behavioral and Brain Sciences, 33(2-3), 137-193.

Cronbach, L. J. (1957). The two disciplines of scientific psychology. American Psychologist, 12(11), 671.

Dalege, J., Borsboom, D., van Harreveld, F., \& van der Maas, H. L. J. (2019). The Attitudinal Entropy (AE) Framework as a General Theory of Individual Attitudes. Psychological Inquiry.

Dalege, J., Borsboom, D., van Harreveld, F., van den Berg, H., Conner, M., \& van der Maas, H. L. J. (2016). Toward a formalized account of attitudes: The Causal Attitude Network (CAN) model. Psychological Review, 123(1), 2-22.

Deary, I. J. (2002). g and cognitive elements of information processing: An agnostic view. In R. J. Sternberg \& E. L. Grigorenko (Eds.), The general factor of intelligence (pp. 151-182). Mahwah: Lawrence Erlbaum.

Deary, I. J., Strand, S., Smith, P., \& Fernandes, C. (2007). Intelligence and educational achievement. Intelligence, 35(1), 13-21.

Dickens, W. T. (2007). What is g? Washington, DC: Brookings Institution.

Dweck, C. S. (1986). Motivational processes affecting learning. American Psychologist, 41(10), 1040-1048. 
Cannon, T. D., \& Keller, M. C. (2006). Endophenotypes in the genetic analyses of mental disorders. Annu. Rev. Clin. Psychol., 2, 267-290.

Edwards, J., \& Bagozzi, R. (2000). On the nature and direction of relationships between constructs and measures. Psychological Methods, 5(2), 155-174.

Epskamp, S., Borsboom, D., \& Fried, E. I. (2018). Estimating psychological networks and their accuracy: A tutorial paper. Behavior Research Methods, 50(1), 195-212.

Epskamp, S., Cramer, A. O., Waldorp, L. J., Schmittmann, V. D., \& Borsboom, D. (2012). Qgraph: Network visualizations of relationships in psychometric data. Journal of Statistical Software, 48(4), 1-18.

Eysenck, H. (2018). Intelligence: A new look. Routledge.

Fisher, C., Hall, D. J., Rakowitz, S., \& Gleitman, L. (1994). When it is better to receive than to give: Syntactic and conceptual constraints on vocabulary growth. In L. Gleitman \& B. Landua (Eds.), The acquisition of the lexicon (pp. 333-375). Cambridge: MIT Press.

Forbes, M. K., Wright, A. G., Markon, K. E., \& Krueger, R. F. (2017). Evidence that psychopathology symptom networks have limited replicability. Journal of Abnormal Psychology, 126(7), 969.

Fortuin, C. M., \& Kasteleyn, P. W. (1972). On the random-cluster model. Physica, 57(4), 536-564.

Friedman, J., Hastie, T., \& Tibshirani, R. (2014). Glasso: Graphical lassoestimation of Gaussian graphical models. Retrieved from https://cran.rproject.org/web/packages/glasso/ (R package version 1.0).

Fulker, D. W., DeFries, J. C., \& Plomin, R. (1988). Genetic influence on general mental ability increases between infancy and middle childhood. Nature, 336(6201), 767-769.

Gardner, H. (1995). Reflections on multiple intelligences: Myths and messages. Phi Delta Kappan, 77(3), 200.

Garlick, D. (2002). Understanding the nature of the general factor of intelligence: The role of individual differences in neural plasticity as an explanatory mechanism. Psychological Review, 109(1), 116-136.

Gathercole, S. E. (1999). Cognitive approaches to the development of short-term memory. Trends in Cognitive Sciences, 3(11), 410-418.

Gibson, J. J. (1986). The ecological approach to visual perception. Hillsdale, NJ: Lawrence Erlbaum Associates, Inc.

Gignac, G. E. (2014). Dynamic mutualism versus $g$ factor theory: An empirical test. Intelligence, 42, 89-97.

Gignac, G. E. (2016). Residual group-level factor associations: Possibly negative implications for the mutualism theory of general intelligence. Intelligence, 55, 69-78. 
Golino, H. F., \& Demetriou, A. (2017). Estimating the dimensionality of intelligence like data using exploratory graph analysis. Intelligence, 62, 54-70.

Gottfredson, L. S. (1998). The general intelligence factor. Scientific American Presents, 9, 24-29.

Haworth, C. M., Wright, M. J., Luciano, M., Martin, N. G., de Geus, E. J., van Beijsterveldt, C. E. M. ,Bartels, M., Posthuma, D., Boomsma, D. I., Davis, O. S. P., Kocas Y, Corley, R. P. , DeFries, J. C., Hewitt, J. K., Olson, R. K., Rhea, S.-A., Wadsworth, S. J., Iacono, W. G., McGue, M., Thompson, L. A., Hart, S. A, Petrill, S. A., Lubinski, D., \& Plomin, R. (2010). The heritability of general cognitive ability increases linearly from childhood to young adulthood. Molecular psychiatry, 15(11), 1112.

Hofman, A.D., Kievit, R., Stevenson, C., Molenaar, D., Visser, I, \& van der Maas, H. L. J. (2018). The dynamics of learning maths: A comparison of the g-factor and mutualistic network theory. PsyArxiv preprint. Retrieved from https://doi.org/10.31219/osf.io/xa2ft

Honzik, M. P. (1983). Measuring mental abilities in infancy. New York: Plenum Press.

Jensen, A. R. (1998). The $g$ factor: The science of mental ability. Westport CT US: Praeger Publishers / Greenwood Publishing Group Inc.

Jonas, K. G., \& Markon, K. E. (2016). A descriptivist approach to trait conceptualization and inference. Psychological Review, 123(1), 90.

Jung, R. E., \& Haier, R. J. (2007). The Parieto-Frontal Integration Theory (P-FIT) of intelligence: converging neuroimaging evidence. Behavioral and Brain Sciences, 30(2), 135-154.

Kan, K. J., van der Maas, H. L., \& Kievit, R. A. (2016). Process overlap theory: Strengths, limitations, and challenges. Psychological Inquiry, 27(3), 220-228

Kan, K. J., Wicherts, J. M., Dolan, C. V., \& van der Maas, H. L. (2013). On the nature and nurture of intelligence and specific cognitive abilities: The more heritable, the more culture dependent. Psychological Science, 24(12), 2420-2428.

Kan, K. J., van der Maas, H. L. J. \& Levine (submitted). Extended psychometric network analyses on cognitive data from a communitybased sample reveal evidence against $g$ in favor of mutualism. Manuscript submitted for publication.

Kievit, R. A., Hofman, A. D., \& Nation, K. (submitted). Mutualistic coupling between vocabulary and reasoning in young children: A replication and extension of Kievit et al. 
Kievit, R. A., Davis, S. W., Griffiths, J., Correia, M. M., \& Henson, R. N. (2016). A watershed model of individual differences in fluid intelligence. Neuropsychologia, 91, 186-198.

Kievit, R. A., Lindenberger, U., Goodyer, I. M., Jones, P. B., Fonagy, P., Bullmore, E. T., The Neuroscience in Psychiatry Network \& Dolan, R. J. (2017a). Mutualistic coupling between vocabulary and reasoning supports cognitive development during late adolescence and early adulthood. Psychological Science, 28(10), 1419-1431.

Kievit, R.A., Brandmaier, A., Ziegler, G., van Harmelen, A. L., de Mooij, S. M. M., Moutoussis, M., Goodyer I., Bullmore E., Jones, P. B., Fonagy, P., Lindenberger, U., Dolan, R. J., \& NSPN Consortium (2017b). Developmental cognitive neuroscience using Latent Change Score models: A tutorial and applications. Developmental Cognitive Neuroscience.

Kovacs, K., \& Conway, A. R. (2016). Process overlap theory: A unified account of the general factor of intelligence. Psychological Inquiry, 27(3), 151-177.

Krijnen, W. P. (2004). Positive loadings and factor correlations from positive covariance matrices. Psychometrika, 69(4), 655-660.

Lumsden, J. (1976). Test theory. Annual review of psychology, 27(1), 251280.

May, R. M. (1973). Stability and complexity in model ecosystems. Princeton: Princeton University Press.

McArdle, J. J., (2001). A latent difference score approach to longitudinal dynamic structural analysis. In Structural Equation Modeling: Present and Future. A Festschrift in Honor of Karl Jöreskog, ed. R Cudeck, S du Toit, D. Sörbom. pp. 341-80. Lincolnwood, IL: Sci. Softw. Int.

McArdle, J. J., Ferrer-Caja, E., Hamagami, F., \& Woodcock, R. W. (2002). Comparative longitudinal structural analyses of the growth and decline of multiple intellectual abilities over the life span. Developmental Psychology, 38(1), 115-142.

McCall, R. B., \& Carriger, M. (1993). A meta-analysis of infant habituation and recognition memory performance as predictors of later IQ. Child Development, 64(1), 57-79.

Molenaar, P. C. M., Boomsma, D. I., \& Dolan, C. V. (1993). A third source of developmental differences. Behavior Genetics, 23(6), 519524.

Murray, J. D. (2002). Mathematical biology. I: An introduction (3th ed.). Berlin: Springer Verlag. 
Nisbett, R. E., Aronson, J., Blair, C., Dickens, W., Flynn, J., Halpern, D. F., \& Turkheimer, E. (2012). Intelligence: New findings and theoretical developments. American psychologist, 67(2), 130.

Peng, P, Wang, T, Wang, C, \& Lin, X (2018). A Meta-analysis on the Relation between Fluid Intelligence and Reading/Mathematics: Effects of Tasks, Age, and Social Economics Status. Psychological Bulletin, in press.

Pinker, S. (1994). How could a child use verb syntax to learn verb semantics? In L. Gleitman \& B. Landua (Eds.), The Acquisition of the Lexicon (pp. 377-410). Cambridge: MIT Press.

Plomin, R., \& von Stumm, S. (2018). The new genetics of intelligence. Nature Reviews Genetics, 19(3), 148.

Plsek, P. E., \& Greenhalgh, T. (2001). Complexity science: The challenge of complexity in health care. BMJ: British Medical Journal, 323(7313), 625.

Prakash, S., \& de Roos, A. M. (2004). Habitat destruction in mutualistic metacommunities. Theoretical Population Biology, 65(2), 153-163.

Raykov, T., \& Marcoulides, G. A. (2006). On multilevel model reliability estimation from the perspective of structural equation modeling. Structural Equation Modeling, 13(1), 130-141.

Sachisthal S. M., Jansen B. R., Peetsma T. D., Dalege J., van der Maas, H. L. J., \& Raijmakers, M. E. J. (in press). Introducing a Science Interest Network Model to Reveal Country Differences. Journal of Educational Psychology.

Savi, A. O., Marsman, M., van der Maas, H. L. J., \& Maris, G. K. J. (2018). The wiring of intelligence. PsyArxiv preprint. Retrieved from https://doi.org/10.31234/osf.io/32wr8.

Schmidhuber, J. (2015). Deep learning in neural networks: An overview. Neural networks, 61, 85-117.

Schwaighofer, M., Fischer, F., \& Bühner, M. (2015). Does working memory training transfer? A meta-analysis including training conditions as moderators. Educational Psychologist, 50(2), 138-166.

Shahabi, S. R., Abad, F. J., \& Colom, R. (2018). g, mutualism, and development: Cross-sectional evidence from Iranian school children. Personality and Individual Differences, 135, 222-228.

Siegler, R. S., \& Alibali, M. W. (2004). Children's thinking (4th ed.). Upper Saddle River, NJ: Prentice Hall.

Sternberg, R. J. (1988). A three-facet model of creativity. In R. J. Sternberg (Ed.), The nature of creativity: Contemporary psychological perspectives (pp. 125-147). New York, NY, US: Cambridge University Press. 
Strogatz, S. H. (2018). Nonlinear dynamics and chaos: With applications to physics, biology, chemistry, and engineering. CRC Press.

Thomson, G. (1951). The factorial analysis of human ability (5th ed.). London: University of London Press.

Thorndike, R. L. (1927). The measurement of intelligence. New York: Teachers College.

van Bork, R., Epskamp, S., Rhemtulla, M., Borsboom, D., \& van der Maas, H. L. J. (2017). What is the p-factor of psychopathology? Some risks of general factor modeling. Theory \& Psychology, 27(6), 759773.

van de Leemput, I. A., Wichers, M., Cramer, A. O., Borsboom, D., Tuerlinckx, F., Kuppens, P., ... \& Derom, C. (2014). Critical slowing down as early warning for the onset and termination of depression. Proceedings of the National Academy of Sciences, 111(1), 87-92.

van der Maas, H. L. J, Kan, K. J., Marsman, M., \& Stevenson, C. E. (2017). Network models for cognitive development and intelligence. Journal of Intelligence, 5(2), 16.

van der Maas, H. L. J., \& Kan, K. J. (2016). Comment on "Residual group-level factor associations: Possibly negative implications for the mutualism theory of general intelligence" by Gilles E. Gignac (2016). Intelligence, 57, 81-83.

van der Maas, H. L. J., Dolan, C. V., Grasman, R. P., Wicherts, J. M., Huizenga, H. M., \& Raijmakers, M. E. (2006). A dynamical model of general intelligence: The positive manifold of intelligence by mutualism. Psychological Review, 113(4), 842.

van der Maas, H. L. J., Kan, K. J., \& Borsboom, D. (2014). Intelligence is what the intelligence test measures. Seriously. Journal of Intelligence, 2(1), 12-15.

van der Maas, H. L. J., Kan, K., Hofman, A., \& Raijmakers, M. (2014). Dynamics of development: A complex systems approach. In P. C. M. Molenaar, R. M. Lerner, \& K. M. Newell (Eds.), Handbook of developmental systems theory and methodology (pp. 270-286). New York, NY: Guilford Press.

van Geert, P. (1991). A dynamic systems model of cognitive and language growth. Psychological Review, 98(1), 3-53.

Weisbuch, G. (2018). Complex systems dynamics. CRC Press. 